\title{
A REFINED REVERSE ISOPERIMETRIC INEQUALITY IN THE PLANE
}

\section{Shengliang Pan, Xueyuan Tang And XiaOyu Wang}

Abstract. It is proved that if $\gamma$ is a closed strictly convex curve in the plane with length $L$ and area $A$, then

$$
L^{2} \leqslant 4 \pi A+2 \pi|\tilde{A}|,
$$

with equality holding if and only if $\gamma$ is a circle, where $\tilde{A}$ denotes the oriented area enclosed by the locus of curvature centers of $\gamma$.

Mathematics subject classification (2010): 52A38, 52A40.

Keywords and phrases: Minkowski's support function, locus of centers of curvature, integral of radius of curvature, reverse isoperimetric inequality.

\section{REFERENCES}

[1] K. BALL, Volume ratios and a reverse isoperimetric inequality, J. London Math. Soc., 44 (1991), 351-359.

[2] C. BANDLE, Isoperimetric Inequalities and Applications, Pitman, Boston, 1980.

[3] V. Blasıö, The isoperimetric problem, Amer. Math. Monthly, 112 (2005), 526-566.

[4] T. Bonnesen \& W. Fenchel, Theorie der Convexen Körper, Chelsea Publishing, New York, 1948.

[5] Yu D. Burago \& V. A. Zelgaller, Geometric Inequalities, Berlin: Springer-Verlag, 1988.

[6] I. Chavel, Isoperimetric Inequalities, Differential Geometric and Analytic Perspectives, Combridge University Press, 2001.

[7] F. EDLER, Vervollständigung der Steinerschen elementargeometrischen Beweise für den Satz, das der Kreis grösseren Flächeninhalt besitzt als jede andere ebene Figur gleich grossen Umfangs, Nachr. Ges. Wiss. Göttingen, 1882, 73-80. [translated into French and printed in Bull. Sci. Math., 7, 2 (1883), 198-204].

[8] R. J. GARDNer, Brunn-Minkowski inequality, Bull. Amer. Math. Soc., 39 (2002), 355-405.

[9] M. GREEN \& S. OSHER, Steiner polynomials, Wulff flows, and some new isoperimetric inequalities for convex plane curves, Asia J. Math., 3 (1999), 659-676.

[10] R. HOWARD \& A. TREIGERGS, A reverse isoperimetric inequality, stability and extremal theorems for plane curves with bounded curvature, Rocky Mountain J. Math., 25 (1995), 635-684.

[11] C. C. Hsiung, A First Course in Differential Geometry, Pure \& Applied Math., Wiley, New York, 1981.

[12] D. A. Klain, An error estimate for the isoperimetric deficit, Illinois J. Math., 49 (2005), 981-992.

[13] G. LAWLOR, A new area-maximization proof for the circle, Math. Intelligencer, 20 (1998), 29-31.

[14] R. Osserman, The isoperimetric inequalities, Bull. Amer. Math. Soc., 84 (1978), 1182-1238.

[15] R. Osserman, Bonnesen-style isoperimetric inequalities, Amer. Math. Monthly, 86 (1979), 1-29.

[16] S. L. PAN, A note on the general plane cueve flows, J. Math. Study, 33 (2000), 17-26.

[17] S. L. PAN \& J. N. YANG, On a non-local perimeter-preserving curve evolution problem for convex plane curves, Manuscripta Math., 127 (2008), 469-484.

[18] S. L. PAN \& H. ZhANG, A reverse isoperimetric inequality for closed strictly convex plane curves, Beiträge zur Algebra und Geometrie, 48 (2007), 303-308.

[19] L. A. Santaló, Integral geometry and Geometric Probability, Addison-Wesley Publishing Co., Reading, Mass.-London-Amsterdam, 1976.

[20] R. SchneIder, Convex Bodies: the Brunn-Minkowski Theory, Cambridge University Press, Cambridge-New York, 1993. 
[21] J. STEINER, Sur le maximum et le minimum des figures dans le plan, sur la sphère, et dans l'espace en général, I and II, J. Reine Angew. Math. (Crelle), 24 (1842), 93-152 and 189-250.

[22] G. TAlenti, The standard isoperimetric inequality, in "Handbook of Convex Geometry", Vol. A, edited by P. M. Gruber and J. M. Wills, pp. 73-123, Amsterdam: North-Halland, 1993. 